Proceedings of 6th Workshop on Quantum Chaos and Localisation Phenomena, Warsaw, May 24-26, 2013

\title{
Scattering from a Ring Graph — A Simple Model for the Study of Resonances
}

\author{
D. WALTNER ${ }^{a}$ AND U. SMilAnSkY ${ }^{a, b}$ \\ ${ }^{a}$ Department of Physics of Complex Systems, Weizmann Institute of Science, Rehovot, Israel \\ ${ }^{b}$ School of Mathematics, Cardiff University, Cardiff, Wales, UK
}

\begin{abstract}
Scattering from the very simple ring graph is shown to display several basic features which underlie the complex (chaotic) phenomena observed in scattering from more complex graphs. In particular we demonstrate the appearance of arbitrarily narrow resonances - the "topological resonances" which are directly linked to the existence of cycles. We use the ring graph to study the response of such resonances to perturbations induced by a time-dependent random noise.
\end{abstract}

DOI: $10.12693 /$ APhysPolA.124.1087

PACS: 05.40.Ca, 05.45.Mt, 03.65.Sq, 02.10.Ox

\section{Introduction}

Graphs were introduced to the quantum chaos community as a paradigm model which is simple to study both analytically and numerically, yet displaying the complex features which characterize quantum chaotic dynamics [1]. The relevant developments on the subject are summarized in review articles, such as e.g., $[2,3]$, and a recent book [4]. Graphs consist of vertices connected by edges (bonds). Associating a length (metric) to the bonds, and adding semi-infinite leads from some vertices to infinity define the geometry of the metric graph on which scattering can be studied. The quantum (wave) dynamics is described by the Schrödinger operator on the graph: On each bond or lead the wave function satisfies the one-dimensional wave equation, which, in the absence of a potential, can be explicitly written $\Psi_{k}(x)=A(k) \mathrm{e}^{\mathrm{i} k x}+B(k) \mathrm{e}^{-\mathrm{i} k x}$, where $k$ is the wave number. At the vertices, appropriate boundary conditions are imposed so that the resulting operator is self-adjoint and the coefficients $A(k), B(k)$ for the bonds and leads can be uniquely computed. Here we shall assume the Neumann boundary conditions throughout, that is, at each vertex $v$, which is connected to $d$ bonds and leads, the wave function is continuous and the sum of the outgoing derivatives vanishes

$$
\sum_{e=1}^{d} \frac{\mathrm{d} \Psi_{e}}{\mathrm{~d} x_{e}}(v)=0
$$

Since the study of chaotic scattering on graphs was initiated [5], the subject was investigated intensively and the scattering resonances and their statistics is a recurring research theme (see e.g., the recent papers $[6,7]$ and the literature cited therein). In particular, very narrow resonances turn out to be quite sensitive to perturbations of the underlying system. In Ref. [8] this was shown by introducing a weak nonlinear perturbation on one bond of a graph. We consider in these notes another perturbation a graph might be exposed to. Normally a graph cannot be considered as isolated from its environment but subject to time dependent external effects (noise). We will show its effect on the shape of the resonances in the system.

The outline of this article is as follows. In the next section we introduce the ring graph and compute the wave functions on the bonds and leads. We provide explicit expressions for the scattering matrix elements, and for the scattering resonances. Finally we introduce a random, time-dependent perturbation on the graph and explain its effect on the resonances.

\section{The ring graph}

We consider here the ring graph shown in Fig. 1. A scattering solution with a wave number $k$ on the ring graph, with an incoming wave in the lead " 1 " and only outgoing wave in " 4 ", and satisfying the boundary conditions at the vertices can be written down explicitly. It takes the following form on each of the bonds:

$$
\begin{array}{ll}
1: & \Psi_{k}(x)=\mathrm{e}^{\mathrm{i} k x}+R(k) \mathrm{e}^{-\mathrm{i} k x}, \\
2: & \Psi_{k}(x)=A(k) \mathrm{e}^{\mathrm{i} k x}+B(k) \mathrm{e}^{-\mathrm{i} k x}, \\
3: & \Psi_{k}(x)=C(k) \mathrm{e}^{\mathrm{i} k x}+D(k) \mathrm{e}^{-\mathrm{i} k x}, \\
4: & \Psi_{k}(x)=T(k) \mathrm{e}^{\mathrm{i} k x} .
\end{array}
$$

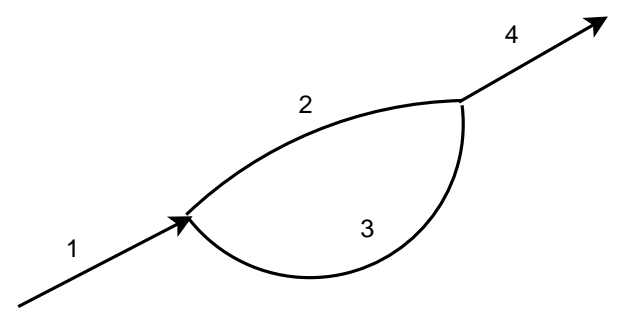

Fig. 1. The ring graph. The two inner bonds labeled by "2" and " 3 " form a circle, connected to the two leads " 1 " and " 4 ".

Denoting by $L_{1}$ and $L_{2}$ the lengths of the bonds " 2 " and ' 3 ", we get, 


$$
\begin{aligned}
& T(k)=\frac{8 \mathrm{i}\left(\sin k L_{2}+\sin k L_{1}\right)}{\mathcal{D}(k)}, \\
& R(k)=\frac{2 \cos k\left(L_{2}-L_{1}\right)+6 \cos k\left(L_{2}+L_{1}\right)-8}{\mathcal{D}(k)}, \\
& A(k)=\frac{2\left[\mathrm{e}^{\mathrm{i} k\left(L_{2}-L_{1}\right)}+2-3 \mathrm{e}^{-\mathrm{i} k\left(L_{1}+L_{2}\right)}\right]}{\mathcal{D}(k)}, \\
& B(k)=\frac{2\left[\mathrm{e}^{\mathrm{i} k\left(L_{1}+L_{2}\right)}+\mathrm{e}^{-\mathrm{i} k\left(L_{2}-L_{1}\right)}-2\right]}{\mathcal{D}(k)}, \\
& C(k)=\frac{2\left[2+\mathrm{e}^{-\mathrm{i} k\left(L_{2}-L_{1}\right)}-3 \mathrm{e}^{-\mathrm{i} k\left(L_{1}+L_{2}\right)}\right]}{\mathcal{D}(k)}, \\
& D(k)=\frac{2\left[\mathrm{e}^{\mathrm{i} k\left(L_{1}+L_{2}\right)}-2+\mathrm{e}^{\mathrm{i} k\left(L_{2}-L_{1}\right)}\right]}{\mathcal{D}(k)} .
\end{aligned}
$$

Here

$$
\begin{aligned}
& \mathcal{D}(k)=8-\mathrm{e}^{\mathrm{i} k\left(L_{1}+L_{2}\right)}+\mathrm{e}^{\mathrm{i} k\left(L_{2}-L_{1}\right)}+\mathrm{e}^{\mathrm{i} k\left(L_{1}-L_{2}\right)} \\
& \quad-9 \mathrm{e}^{-\mathrm{i} k\left(L_{1}+L_{2}\right)} .
\end{aligned}
$$

The transmission and reflection amplitudes $T(k)$ and $R(k)$ are two entries of the (two-dimensional) scattering matrix for the ring graph. The other two (corresponding to an incoming wave from lead " 4 ") can be written down in a similar way. Resonances are identified as the poles of the scattering matrix in the complex $k$-plane. The real parts of these $k$-values determine the wave number where the resonances occur and the imaginary parts are proportional to the resonance widths. The poles are identified by the zeros of $\mathcal{D}(k)$ in Eq. (4) provided that the residues at these zeros do not vanish. When this happens, the spectrum of the graph is no more exclusively continuous, but proper eigenvalues with normalizable wave functions appear at discrete $k$ values. These states which are embedded in the continuum play an important role in the present discussion.

The structure of the resonances of the ring graph in Fig. 1 depends on the ratio of the lengths $L_{1}$ and $L_{2}$.

We start with considering equal lengths $L_{1}=L_{2}=$ $2 L_{0}$. The zeros of $\mathcal{D}(k)$ in this case are located at $\kappa_{n}=n \pi /\left(2 L_{0}\right)-\mathrm{i} \ln 3 /\left(2 L_{0}\right)$ and $k_{n}=n \pi /\left(2 L_{0}\right)$ with $n \in \mathbb{N}_{0}$. The first set of zeros corresponds to complex poles of the scattering matrix, and hence to a set of broad resonances with a $k$ independent width $\ln 3 /\left(2 L_{0}\right)$. They are the resonances which are usually called "shape resonances" in scattering theory, and are seen as broad peaks in the blue curve in Fig. 2 where the transmission spectrum $|T(k)|^{2}$ is plotted. The set of real zeros $k_{n}$ corresponds to states embedded in the continuum, with wave functions which vanish at the two leads and therefore also at the two vertices. They cannot be noticed in a scattering experiment where only the transmission and reflection are measured. Let us note that the states embedded in the continuum appear for any ring graph with rationally related lengths. This is a set of measure zero but its presence affects also scattering from graphs with irrational ratios as will be seen below.

Indeed, consider the case $L_{1}=2 L_{0}$ and $L_{2}=2 L_{0}(1+\varepsilon)$

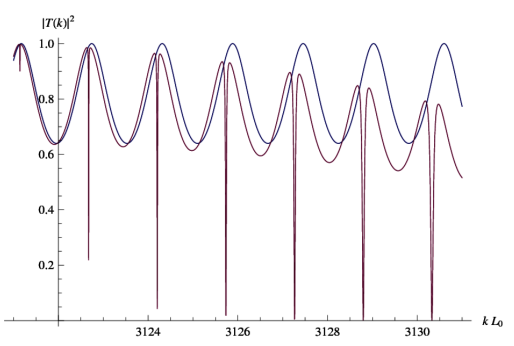

Fig. 2. In blue the transmission spectrum $|T(k)|^{2}$ obtained for the graph in Fig. 1 for equal lengths of the bonds " 2 " and " 3 ", $L_{1}=L_{2}=2 L_{0}$ is shown. The violet plot is obtained for $L_{1}=2 L_{0}$ and $L_{1}=(2+\pi / 30) L_{0}$.

with irrational $\varepsilon \ll 1$. The transmission spectrum for $\varepsilon=\frac{\pi}{15}$ is shown in the red curve in Fig. 2 as an illustration. As long as $n \varepsilon$ is small, the shape resonances which dominated the scene for $L_{1}=L_{2}$ are only slightly affected, but a new set of sharp resonances appear, and their position approximately coincides with that of the shape resonances. This can be easily explained by considering $\varepsilon$ as a small perturbation. Then, for $k \approx n \pi /\left(2 L_{0}\right)$ there are two poles in the complex plane instead of one. The poles which produce the shape resonances move only slightly. The zeros of $\mathcal{D}(k)$ which corresponded to bound states are moving away from the real axis, with small imaginary parts, leading to the very narrow dips visible within the broad resonances in Fig. 2. In order to obtain a better understanding of the location of resonances in the complex plane we analyze the zeros of $\mathcal{D}(k)$ with $\Sigma=L_{1}+L_{2}$ and $\delta=L_{1}-L_{2}$ in the limit $n \delta \ll \Sigma$. Writing the zeros of $\mathcal{D}(k)$ as $k=k^{\prime}+\mathrm{i} \gamma$ we get the two equations,

$$
\begin{aligned}
& 8\left[1-\cos \left(k^{\prime} \Sigma\right) \mathrm{e}^{\gamma \Sigma}\right]-\cos \left(k^{\prime} \Sigma\right)\left(\mathrm{e}^{\gamma \Sigma}+\mathrm{e}^{-\gamma \Sigma}\right) \\
& +\cos \left(k^{\prime} \delta\right)\left(\mathrm{e}^{-\gamma \delta}+\mathrm{e}^{\gamma \delta}\right)=0
\end{aligned}
$$

and

$$
\begin{aligned}
& 8 \sin \left(k^{\prime} \Sigma\right) \mathrm{e}^{\gamma \Sigma}-\sin \left(k^{\prime} \Sigma\right)\left(\mathrm{e}^{-\gamma \Sigma}-\mathrm{e}^{\gamma \Sigma}\right) \\
& \quad+\sin \left(k^{\prime} \delta\right)\left(\mathrm{e}^{-\gamma \delta}-\mathrm{e}^{\gamma \delta}\right)=0 .
\end{aligned}
$$

With the ansatz $k^{\prime} \Sigma=2 \pi n+\alpha, n \delta / \Sigma \ll 1, \gamma \Sigma=\beta$ and $\alpha, \beta \ll 1$ we find that to order $(\delta / \Sigma)^{2}, \alpha=0$ and $\beta=-\frac{1}{8}(2 \pi n \delta / \Sigma)^{2}$. Thus, the resonances may have an arbitrarily narrow width.

Similarly, denoting the shifts of the shape resonance poles as $k^{\prime} \Sigma=2 \pi n+\alpha$ and $\gamma \Sigma=-2 \ln 3+\beta$, we get $\alpha=\pi n \ln 3(\delta / \Sigma)^{2}$ and $\beta=-\left[(2 \ln 3)^{2}-(2 \pi n)^{2}\right](\delta / \Sigma)^{2} / 8$ which are small changes compared to $k^{\prime} \Sigma=2 \pi n$ and $\gamma \Sigma=-2 \ln 3$.

The mechanism which produces the narrow resonances is analogous to the one producing the Feshbach resonances in scattering theory [9]. Here the parameter which is varied and which turns the bound state into a resonance is the ratio of the two arms of the cycle in the graph. However, the full set of resonances appears only for an irrational ratio - a distinction which is a peculiarity of the graph system (but is known in various other instances in quantum chaos, such as e.g., the Floquet spectrum of the periodically kicked rotor). 
Another way to generate the narrow resonances is to start from a symmetric ring, and introduce a $\delta$ potential $V(x)=\alpha \delta\left(x-x_{0}\right)$ at an arbitrary point $x_{0}$ on one of the ring bonds. As long as $x_{0}$ is not a rational fraction of $L_{0}$, this perturbation with arbitrarily small $\alpha$ will turn all the states embedded in the continuum into resonances with arbitrarily narrow width. This is another way to generate the same Feshbach resonances, and this is illustrated in Fig. 3.

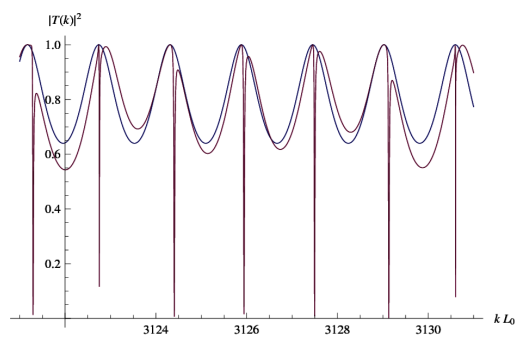

Fig. 3. The transmission through a symmetric ring graph perturbed by a delta potential placed at an irrational point $x_{0}$ on bond "2", for $\alpha=0$ (blue) and $m \alpha /\left(\hbar^{2} k\right)=0.25$ (violet).

Adding a $\delta$ potential for a ring graph with irrational ratio of the bond lengths does not change the resonance structure in a quantitative way, and if $\alpha$ is small enough it only shifts the narrow resonances and does not affect the broad structures which are due to the "shape" resonances. The $\alpha$ values needed to achieve a shift here are much smaller than the ones needed for the shape resonances in Fig. 3.

As a final remark, let us note that the appearance of narrow resonances as discussed above is a general phenomenon which occurs in any graph with cycles, and it is the origin of the topological resonances discussed in [7].

\section{Transmission through a ring graph perturbed by a random time dependent perturbation}

We analyze finally the effect of time dependent perturbations on the graph. We therefore add to the system on bond " 2 " a delta potential located at a time dependent position $\gamma(t)$ centered at an arbitrary point $x_{0}$ with $\gamma(t) \ll\left(L_{1}-x_{0}\right):$

$$
V(x, t)=\alpha \delta\left\{x-\left[x_{0}+\gamma(t)\right]\right\} .
$$

Our purpose is to study the effect of the random noise on the transmission through the ring for an incident wave with $k$ values where the unperturbed graph has narrow resonances.

We calculate [10] the effect of $V(x, t)$ on the current density perturbatively up to second order in $\alpha$. The time evolution of the wave function $\Psi(x, t)$ is determined by the time dependent Schrödinger equation

$$
\mathrm{i} \hbar \frac{\partial}{\partial t} \Psi(x, t)=-\frac{\hbar^{2}}{2 m} \frac{\partial^{2}}{\partial x^{2}} \Psi(x, t)+V(x, t) \Psi(x, t) .
$$

We obtain an expansion $\Psi(x, t)=\Psi^{(0)}(x, t)+$ $\alpha \Psi^{(1)}(x, t)+\alpha^{2} \Psi^{(2)}(x, t)+\ldots$ by recursive solution of the latter equation that we use to calculate the current density

$$
j(x, t)=\frac{\hbar}{2 m \mathrm{i}}\left[\Psi^{*}(x, t) \frac{\partial}{\partial x} \Psi(x, t)-\text { c.c. }\right]
$$

with the expansion $j(x, t)=j^{(0)}(x, t)+\alpha j^{(1)}(x, t)+$ $\alpha^{2} j^{(2)}(x, t)+\ldots$ and c.c. denoting the complex conjugate. The unperturbed current density $j^{(0)}(x, t)$ is given in terms of the wave function by

$$
\begin{gathered}
j^{(0)}(x, t)=\frac{\hbar}{2 m \mathrm{i}}\left[\Psi^{(0) *}(x, t) \frac{\partial}{\partial x} \Psi^{(0)}(x, t)-\text { c.c. }\right] \\
=\frac{\hbar k}{m}|T(k)|^{2}
\end{gathered}
$$

and the first two leading order corrections

$$
\begin{gathered}
j^{(1)}(x, t)=\frac{\hbar}{2 m \mathrm{i}}\left[\Psi^{(0) *}(x, t) \frac{\partial}{\partial x} \Psi^{(1)}(x, t)\right. \\
\left.+\Psi^{(1) *}(x, t) \frac{\partial}{\partial x} \Psi^{(0)}(x, t)-\text { c.c. }\right]
\end{gathered}
$$

and

$$
\begin{aligned}
& j^{(2)}(x, t)=\frac{\hbar}{2 m \mathrm{i}}\left[\Psi^{(1) *}(x, t) \frac{\partial}{\partial x} \Psi^{(1)}(x, t)\right. \\
& +\Psi^{(0) *}(x, t) \frac{\partial}{\partial x} \Psi^{(2)}(x, t) \\
& \left.+\Psi^{(2) *}(x, t) \frac{\partial}{\partial x} \Psi^{(0)}(x, t)-\text { c.c. }\right] .
\end{aligned}
$$

The resulting expressions for the current density depend on $\gamma\left(t^{\prime}\right)$ with $t^{\prime}<t$. We are however interested not in the results for a specific realization of the noise introduced by the fluctuating position $\gamma(t)$, but in its average behavior. We therefore assume that $\gamma(t)$ performs a Brownian motion (diffusion with constant diffusion constant and no drift) and average $\gamma(t)$ with the Wiener measure of Brownian motion. Then the squared average of $\gamma(t)$ increases linearly with time [11]:

$$
\left\langle\gamma^{2}(t)\right\rangle=\sigma^{2} t
$$

with the proportionality constant $\sigma^{2}$ characterizing this increase.

We can then study how the considered perturbation affects the current density in dependence of the two parameters $\alpha$ and $\sigma^{2}$. We obtain in general that the effect of the perturbation increases with decreasing width of the resonance as already reported for a static perturbation above. The corrections linear in $\alpha$ to $j(x, t)$ possess only two different forms: one for $\sigma^{2} m / \hbar=0$, i.e. a static perturbation and one for $\sigma^{2} m / \hbar>0$. The dependence of $j^{(2)}(x, t)$ on $\sigma^{2}$ is more involved. We show in Fig. 4 the dependence of $|T(k)|^{2}$ calculated up to second order in $\alpha$ on the parameters $\alpha$ and $\sigma^{2}$ for equal lengths of the bonds " 2 " and " 3 ". Note that within our perturbative analysis the very narrow resonances shown in Fig. 3 cannot be obtained. In Fig. 5 for rationally independent lengths of the bonds " 2 " and " 3 " the analogous plots are presented. 

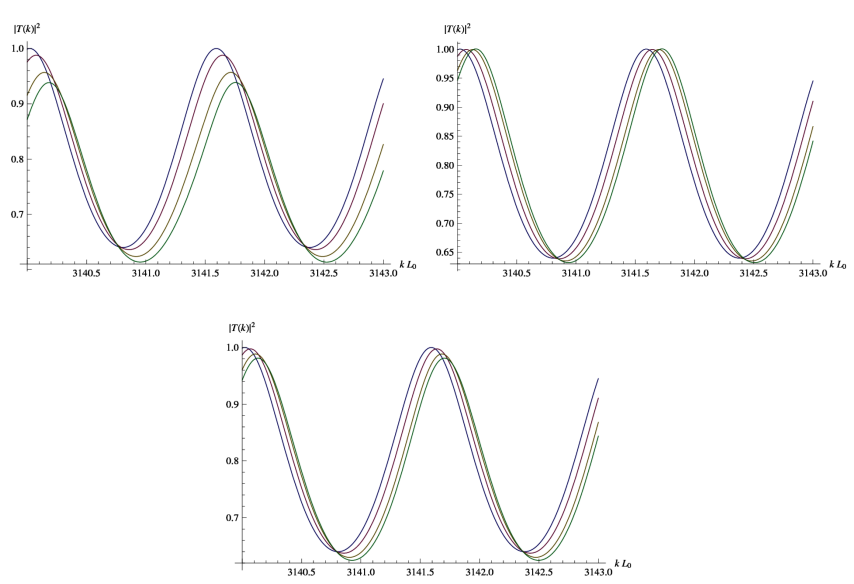

Fig. 4. The $k$-dependence of the transmission for shape resonances. In the first plot we consider $\sigma^{2} m / \hbar=10^{-7}$, in the second $\sigma^{2} m / \hbar=0.1$ and in the third $\sigma^{2} m / \hbar=$ $10^{5}$. In each plot the different curves show the transmission obtained for $m \alpha /\left(\hbar^{2} k\right)=0$ (blue), $m \alpha /\left(\hbar^{2} k\right)=0.2$ (violet), $m \alpha /\left(\hbar^{2} k\right)=0.4$ (yellow), and $m \alpha /\left(\hbar^{2} k\right)=0.5$ (green).
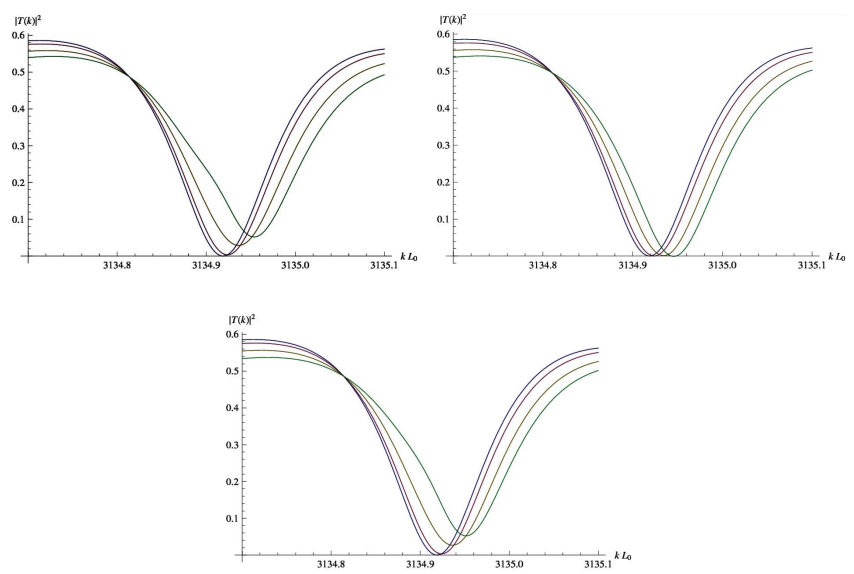

Fig. 5. The $k$-dependence of the transmission considering a very narrow (topological) resonance. We consider $\sigma^{2} m / \hbar=10^{-9}$ in the first plot, $\sigma^{2} \mathrm{~m} / \hbar=10^{-3}$ in the second and $\sigma^{2} m / \hbar=10^{7}$ in the third. The different curves in each plot are obtained for the unperturbed situation $\left(\alpha=0\right.$, blue), $m \alpha /\left(\hbar^{2} k\right)=0.02$ (violet), $m \alpha /\left(\hbar^{2} k\right)=0.06$ (yellow), and $m \alpha /\left(\hbar^{2} k\right)=0.1$ (green).

In conclusion, we observe that additionally to the above mentioned shift there is also a broadening of the resonance in the regimes $\hbar / m \gg \sigma^{2}$ and $\hbar / m \ll \sigma^{2}$.

\section{Conclusions}

The transmission through the ring graph was analyzed assuming various length ratios and perturbations. We have shown that the ring graph displays interesting features such as the occurrence of very long lived (narrow) resonances, and therefore it can be used as a convenient model for understanding some of the mechanisms which bring about chaotic scattering on graphs. We then studied the effect of random noise on the transmission through the graph, by modeling the coupling of the graph to a noisy "environment" by a randomly moving $\delta$ potential on one of the ring bonds.

In many systems of interest, the unavoidable coupling of the system to the environment [12] needs to be taken into account. This was the case in e.g., the study of the effect of noise on the dynamical localization in the Rydberg atoms ionization by periodic electromagnetic fields [13]. We consider the present work as the first step in extending the use of quantum graphs as paradigm model for the study of such randomly perturbed systems.

\section{Acknowledgments}

D.W. would like to thank the Minerva Foundation for financial support. We also acknowledge support by the ISF grant $861 / 11$ (F.I.R.S.T.).

\section{References}

[1] T. Kottos, U. Smilansky, Phys. Rev. Lett. 79, 4794 (1997); Ann. Phys. 274, 76 (1999).

[2] S. Gnutzmann, U. Smilansky, Adv. Phys. 55, 527 (2006).

[3] Analysis on Graphs and Its Applications, Eds. P. Exner, J.P. Keating, P. Kuchment, T. Sunada, A. Teplyaev, Proc. Symp. in Pure Mathematics, American Mathematical Society, Providence 2008.

[4] G. Berkolaiko, P. Kuchment, Introduction to Quantum Graphs, Mathematical Surveys and Monographs, Vol. 186, AMS, Providence 2013.

[5] T. Kottos, U. Smilansky, Phys. Rev. Lett. 85, 968 (2000); J. Phys. A, Math. Gen. 36, 3501 (2003).

[6] Z. Pluhar, H.A. Weidenmüller, Phys. Rev. Lett. 110, 034101 (2013).

[7] S. Gnutzmann, H. Schanz, U. Smilansky, Phys. Rev. Lett. 110, 094101 (2013).

[8] S. Gnutzmann, U. Smilansky, S. Derevyanko, Phys. Rev. A 83, 033831 (2011).

[9] H. Feshbach, Ann. Phys. (New York) 5, 357 (1958).

[10] D. Waltner, Transmission through a Noisy Network, unpublished results.

[11] B. Bodmann, H. Leschke, S. Warzel, in: Path Integrals: Dubna '96, Eds. V.S. Yarunin, M.A. Smondyrev, Dubna 1996, p. 95.

[12] H.J. Carmichael, Statistical Methods in Quantum Optics: Master Equations and Fokker-Planck Equations, Springer, Berlin 1999.

[13] R. Blümel, A. Buchleitner, R. Graham, L. Sirko, U. Smilansky, H. Walther, Phys. Rev. A 44, 4521 (1991). 